Revue internationale P.M.E.

Économie et gestion de la petite et moyenne entreprise

\title{
Les dynamiques d'innovation et de R-D des PMI françaises
}

\section{Jean Bernard et André Torre}

Volume 7, numéro 3-4, 1994

URI : https://id.erudit.org/iderudit/1008422ar

DOI : https://doi.org/10.7202/1008422ar

Aller au sommaire du numéro

Éditeur(s)

Presses de l’Université du Québec

ISSN

0776-5436 (imprimé)

1918-9699 (numérique)

Découvrir la revue

Citer cet article

Bernard, J. \& Torre, A. (1994). Les dynamiques d'innovation et de R-D des PMI françaises. Revue internationale P.M.E., 7(3-4), 19-39.

https://doi.org/10.7202/1008422ar
Résumé de l'article

Cet article vise à analyser les dynamiques innovatrices des PMI françaises à partir d'une étude de données individuelles d'entreprises. On montre que les petites firmes sont rarement plus innovatrices que les grandes, mais que leur comportement reste largement comparable à celui de ces dernières quand elles sont innovantes. Par ailleurs, les PMI innovatrices sont rarement des leaders en matière d'innovation et se caractérisent par une plus grande turbulence dans le domaine de l'activité de R-D. 


\title{
Les dynamiques d'innovation et de R-D des PMI françaises
}

Jean BERNARD*

Université de Nice

André TORRE**

INRA

\section{MOTS CLÉS}

\section{Innovation - R-D - PMI - France - Taille des firmes \\ Types d'innovation - Sources de l'innovation \\ Intensité de la R-D - Innovation sectorielle}

\begin{abstract}
RÉSUMÉ
Cet article vise à analyser les dynamiques innovatrices des $P M I$ françaises à partir d'une étude de données individuelles d'entreprises. On montre que les petites firmes sont rarement plus innovatrices que les grandes, mais que leur comportement reste largement comparable à celui de ces dernières quand elles sont innovantes. Par ailleurs, les PMI innovatrices sont rarement des leaders en matière d'innovation et se caractérisent par une plus grande turbulence dans le domaine de l'activité de R-D.
\end{abstract}

\section{ABSTRACT}

The main goal of this paper is to analyse the innovative behavior of the French SMEs, on the basis of an applied study rested upon desagregated datas. It is shown that small firms have a lower level of technological intensity than large ones but also that the innovative behavior of the French firms is not linked to

* Jean Bernard est professeur à l'Université de Nice Sophia-Antipolis. Adresse : Université de Toulon et du Var, Faculté de sciences économiques et de gestion, B.P. 132 F, 83957 La Garde, France.

** André Torre est directeur de recherches à l'INRA, LRDE (Corte). Adresse : LATAPSES, 25, rue Einstein, Sofia-Antipolis, 06560 Valbonne, France. 
their size. The study reveals too that innovative SMEs rarely perform as leaders in terms of innovation on their market but may be characterized by a greater turbulence in their $R-D$ activities.

\section{RESUMEN}

Este articulo tiene por objectivo de analizar las dinamicas inovadoras de las PyMI francesas a partir de un estudio de los datos individuales de las empresas. Se demuestra que las pequenas firmas son raramente mas inovadoras que las grandes pero que el comportamiento de estas quida ampliamente comparable a el de las ultimas cuando son inovantes. Ademas las PyMI inovadoras son exepcionalmente lideres en tema de inovacion. Se caracterizan por una major turbulencia en lo que toca la actividad de $R-D$.

\section{Introduction}

Alors que les années 60 et 70 ont été marquées par le triomphe des mégacorps et le développement de la concentration industrielle, on voit apparaître au cours de la période suivante de nouveaux «modèles » de production, lesquels se caractérisent par un regain de productivité et de dynamisme de la part des firmes de petite taille. On a employé à ce sujet l'expression «small is beautiful» (Schumacher, 1978) ou encore «second industrial divide» (Piore et Sabel, 1984), en mettant l'accent sur le fait que des modes de production différents de ceux de la grande firme émergeaient au sein des économies industrialisées contemporaines. Ce bouleversement a également eu une influence sur la pensée économique en remettant en cause les thèses de Galbraith (1956) sur la production de masse au profit d'une approche souvent fortement localisée des dynamiques industrielles mis de l'avant par les firmes de petite taille fortement flexibles (Carlsson, 1989). Aujourd'hui, tout en constatant la place croissante occupée par les PME et PMI (définies ici comme les firmes ayant une taille comprise entre 20 et 200 salariés), on s'interroge sur les capacités de ces firmes, sur leur dynamisme, sur leur compétitivité, ou encore sur leur capacité à soutenir une activité d'innovation importante.

Dans le cas français, les PMI représentent une part considérable de l'industrie française, puisqu'en 1989 elles employaient 1,82 million de salariés (soit $53,2 \%$ des effectifs industriels contre $49,4 \%$ en 1986) et représentaient $41 \%$ du chiffre d'affaires global de l'industrie ${ }^{1}$. Par ailleurs, elles réalisent également le quart des exportations industrielles, en particulier dans des secteurs tels que la chimie organique ou la fabrication d'instruments et

1. Ces résultats, ainsi que ceux qui suivent, sont tirés de l'Ouvrage du SESSI (1991). 
de matériel de précision, et supportent $37 \%$ des investissements matériels. Leur poids est particulièrement important dans les secteurs de biens de consommation tels que le travail du bois et l'ameublement, mais également dans les secteurs relevant de la production de minerais non ferreux ou du travail des métaux. Plus de $90 \%$ des PMI françaises sont indépendantes, mais celles qui sont associées à des groupes français ou étrangers possèdent un poids industriel, en termes d'investissement ou de chiffre d'affaires, supérieur à celui des firmes indépendantes. Enfin, les PMI apparaissent comme particulièrement dynamiques, puisque la croissance de leurs investissements $(+7,7 \%)$ est plus forte que celle des grandes entreprises $(+4,3 \%)$ pour la période 1986-1989, alors que leur taux de marge progresse plus lentement. Toutefois, leur capacité d'autofinancement est insuffisante pour assurer un apport de ressources externes diversifiées, si bien qu'elles sont contraintes de faire appel de manière quasi systématique au crédit bancaire (qui leur est offert à un coût plus élevé qu'aux grandes entreprises [GE]) et que leur endettement se révèle particulièrement important.

On constate que l'intensité capitalistique (le rapport entre capital d'exploitation et frais de personnel) des PMI s'est accrue au cours des années 80 , mais de manière parallèle à celle des GE. Toutefois, cette augmentation est surtout permise, il faut le noter, dans le cas des entreprises de petite taille par une faible progression du rendement apparent du travail (Paranque, 1992). Il en résulte qu'il faut maintenant à ces firmes plus de capital qu'en début de période afin de produire une unité de valeur ajoutée, ce qui est peut-être dû à l'effort qu'elles ont consenti en matière d'investissement à partir de 1987. En effet, le taux d'investissement productif (investissement/valeur ajoutée) des PMI françaises s'est fortement accru pendant cette période, en particulier dans la tranche des entreprises de 100 à 500 salariés. Dans le domaine financier, la rentabilité des entreprises, compte tenu du capital engagé, est plus importante chez les petites firmes, mais leur avantage tend à diminuer au cours de la période envisagée. La situation s'est en effet sensiblement modifiée, puisque les GE ont fortement augmenté leur rentabilité grâce à une diminution de la part des charges salariales.

Au-delà de ces aspects généraux, les points qui suivent sont particulièrement centrés sur l'activité technologique des PMI françaises. L'objectif est d'évaluer l'intensité innovatrice des petites firmes et les tendances observées au cours des années récentes. La deuxième partie est consacrée à une étude de la relation entre la taille des entreprises, les dépenses de R-D et l'activité d'innovation. La troisième partie tente d'approcher de manière plus dynamique l'activité innovatrice des PMI, en considérant d'une part les sources de l'innovation et, d'autre part, les intentions des firmes en matière d'innovation dans un avenir rapproché. 


\section{Le poids des PMI et des grandes firmes dans l'activité innovatrice}

La discussion sur la relation entre la taille des entreprises et leur activité en matière de R-D ou d'innovation doit nécessairement faire référence tout d'abord aux hypothèses schumpétériennes (1954), lesquelles peuvent se réduire à deux énoncés principaux :

1. La concentration des entreprises sur un marché constitue un facteur favorable aux investissements de R-D et aux performances en matière d'innovation.

2. La taille des entreprises a un effet positif sur leur activité en matière de R-D et d'innovation.

Cette idée, ultérieurement développée par Galbraith en défense des avantages de la grande firme, repose sur plusieurs arguments, recensés par Cohen et al. (1987) :

- la grande taille favoriserait les projets risqués dans le domaine de l'innovation en permettant la disposition de fonds propres non soumis aux aléas des marchés financiers;

- la production de technologie conduirait à l'obtention d'économies d'échelle;

- le rendement de la R-D serait plus fort quand on peut répartir les coûts fixes liés à l'innovation sur un volume important de ventes;

- la R-D se révélerait plus rentable dans les grandes firmes en raison des effets de complémentarité avec d'autres activités ne relevant pas du domaine de la production (telles que la finance ou la commercialisation).

Les études présentées dans l'enquête de Kamien et Schwartz (1982) montrent que cette hypothèse a rarement été remise en question par les études empiriques réalisées pendant les années 60 et 70 , même si les questions d'unités de référence pertinente ou de différences interindustrielles ont commencé à émerger durant cette période.

L'exploitation de l'enquête réalisée en France en 1991 par le ministère de l'Industrie montre que de très nombreuses PMI appartenant aux secteurs manufacturier et agro-alimentaire se présentent comme innovatrices. Il faut cependant remarquer (ce qui vient confirmer les résultats précédents) que le pourcentage de firmes innovantes augmente avec la taille, que ce soit à l'intérieur même de la catégorie des PMI, ou plus encore si l'on compare PMI et grandes entreprises (tableau 1 et graphiques 1 et 2 ). 
À l'évidence, la majorité des innovations se fait dans les PMI, y compris l'introduction de produits nouveaux pour le marché ou de premières en procédés (tableau 2). Mais en pourcentage, le nombre d'entreprises présentes dans la production de produits nouveaux pour le marché et dans la réalisation de premières de procédés technologiques est beaucoup plus fort dans la catégorie des grandes entreprises (GE) que dans celle des firmes de petite taille. Ce résultat n'est pas surprenant étant donné la grande diversité des PMI. Certaines PMI «défensives » restent à l'écart de l'innovation; d'autres, "offensives», s'efforcent de tirer parti de leur environnement, y compris international, pour innover.

\section{TABleau 1}

Activité innovatrice par classe de taille

(France, 1985-1990, population totale)

\begin{tabular}{l|c|c|c}
\hline Taille & $\begin{array}{c}\text { Nombre de firmes } \\
\text { (en \% colonne) }\end{array}$ & $\begin{array}{c}\text { Innovantes } \\
\text { (en \% ligne) }\end{array}$ & $\begin{array}{c}\text { Non innovantes } \\
\text { (en \% ligne) }\end{array}$ \\
\hline $20-49$ & 39 & 55 & 45 \\
$50-99$ & 21 & 66 & 34 \\
$100-199$ & 13 & 70 & 30 \\
$200-499$ & 13 & 79 & 21 \\
PMI & 86 & 61 & 39 \\
GE & 14 & 89 & 11 \\
Total & $\mathbf{1 0 0}$ & $\mathbf{6 0}$ & $\mathbf{4 0}$ \\
\hline
\end{tabular}

Source : LATAPSES, données enquête innovation SESSI.

\section{TABleaU 2}

Type d'innovation par classe de taille

(France, 1985-1990, pour les seules firmes innovantes)

\begin{tabular}{l|c|c|c|c}
\hline Taille & $\begin{array}{c}\text { Amélioration } \\
\text { de produits } \\
\text { existants } \\
\text { (en \% colonne) }\end{array}$ & $\begin{array}{c}\text { Produits } \\
\text { nouveaux } \\
\text { pour le marché } \\
\text { (en \% colonne) }\end{array}$ & $\begin{array}{c}\text { Amélioration } \\
\text { de procédés }\end{array}$ & $\begin{array}{c}\text { Premières } \\
\text { de procédés } \\
\text { (en \% colonne) }\end{array}$ \\
$\begin{array}{l}\text { technologiques } \\
\text { (en \% colonne) }\end{array}$ \\
\hline $20-49$ & 45 & 41 & 45 & 39 \\
$50-99$ & 21 & 21 & 21 & 21 \\
$100-199$ & 13 & 13 & 13 & 14 \\
$200-499$ & 11 & 11 & 11 & 13 \\
PMI & 90 & 86 & 90 & 87 \\
GE & 10 & 14 & 10 & 13 \\
Total & $\mathbf{1 0 0}$ & $\mathbf{1 0 0}$ & $\mathbf{1 0 0}$ & $\mathbf{1 0 0}$ \\
Nombre de firmes & $\mathbf{8 4 4 4}$ & $\mathbf{5 3 6 5}$ & $\mathbf{8 ~ 1 8 9}$ & $\mathbf{3 3 3 5}$ \\
\hline
\end{tabular}

Source: LATAPSES, données enquête innovation SESSI. 
L'enquête du MESR sur les entreprises ayant une activité de R-D (entendue au sens du manuel de Frascati, c'est-à-dire un chercheur employé à plein temps par année) révèle qu'une proportion non négligeable de PMI françaises font de la R-D, mais là encore, les GE sont plus actives.

\section{TABleau 3}

\section{L'effort de R-D par classe de taille}

(France, 1985-1990)

\begin{tabular}{l|c|c|c}
\hline Taille & $\begin{array}{c}\text { Nombre de firmes } \\
\text { ayant une activité } \\
\text { de R-D (Frascati) } \\
\text { (source MERS 1990) } \\
\text { (en \% colonne) }\end{array}$ & $\begin{array}{c}\text { R-D interne } \\
\text { innovantes } \\
\text { (source SESSI) } \\
\text { (en \% ligne) }\end{array}$ & $\begin{array}{c}\text { R-D du groupe } \\
\text { (source SESSI) } \\
\text { (en ligne) }\end{array}$ \\
\hline $20-49$ & 33 & 40 & 18 \\
$50-99$ & 12 & 51 & 28 \\
$100-199$ & 14 & 62 & 37 \\
$200-499$ & 18 & 72 & 46 \\
PMI & 77 & 48 & 25 \\
GE & 23 & $\mathbf{5 0}$ & $\mathbf{2 8}$ \\
Total & $\mathbf{1 0 0}$ & & \\
Nombre de firmes & $\mathbf{2 7 2 1}$ & & \\
\hline
\end{tabular}

Source: LATAPSES, données enquête recherche MERS et enquête innovation SESSI.

Note: Ces sources ne sont pas directement comparables étant donné que l'enquête MERS porte sur les firmes ayant une activité de R-D au sens de Frascati, et que l'enquête SESSI donne à la R-D un sens beaucoup plus large.

Un examen attentif de la littérature montre qu'il faut attendre les années 80 pour assister à un renouveau de la discussion sur la relation entre taille et activité innovatrice, en particulier avec les travaux empiriques de Scherer (1984) et les nouvelles hypothèses théoriques de Winter (1984). L'étude réalisée par Scherer sur les données US semble indiquer que les corrélations entre taille et intensité innovatrice sont faibles, alors que d'autres recherches appliquées suggèrent que ce sont les firmes de taille intermédiaire qui possèdent la plus faible intensité de R-D. C'est également le cas pour la France, ainsi que le révèle l'article de Cremer et Sirbu (1978) qui montre que la relation entre taille et R-D commence par être décroissante avant de remonter pour les firmes de très grande taille, un résultat voisin étant mis en évidence par Pavitt et al. (1987), qui trouvent en particulier que la part de l'effort dévolu aux innovations de procédés est plus importante dans les grandes firmes. 
GRAPHIQUE 1
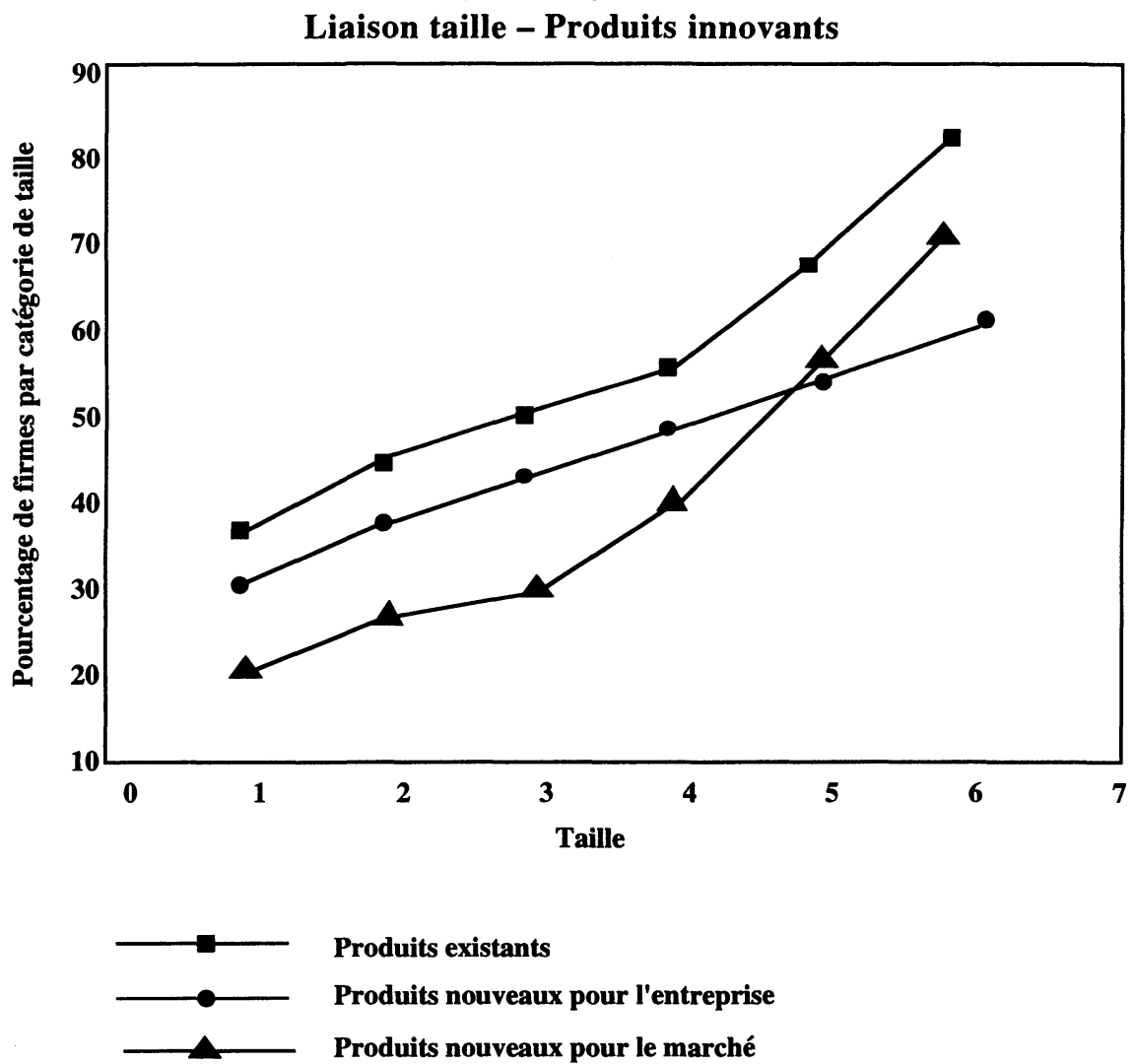

Produits existants

Produits nouveaux pour l'entreprise

Produits nouveaux pour le marché

Des résultats différents sont toutefois obtenus par d'autres auteurs. Ainsi, Acs et Audretsch (1988) observent que l'innovation industrielle diminue quand le degré de concentration augmente. Par ailleurs, Cohen et al. (1987) montrent que la taille croissante de l'établissement est liée de manière positive à la probabilité de procéder à des efforts de R-D. On peut ajouter, avec Kleinknecht (1987), qu'existent des différences significatives à l'intérieur même de la catégorie «PME » (soit des entreprises de moins de 500 employés), le niveau de dépense de R-D variant ici encore selon la taille.

L'hypothèse théorique formulée par Winter (1984) suggère que l'activité innovatrice des petites et des grandes firmes répond à des régimes économiques et technologiques distincts. Il est donc important d'avoir cette différence présente à l'esprit dans tout test comparatif, en particulier en ce qui concerne les types et les modalités d'innovations et de R-D, ainsi que les 

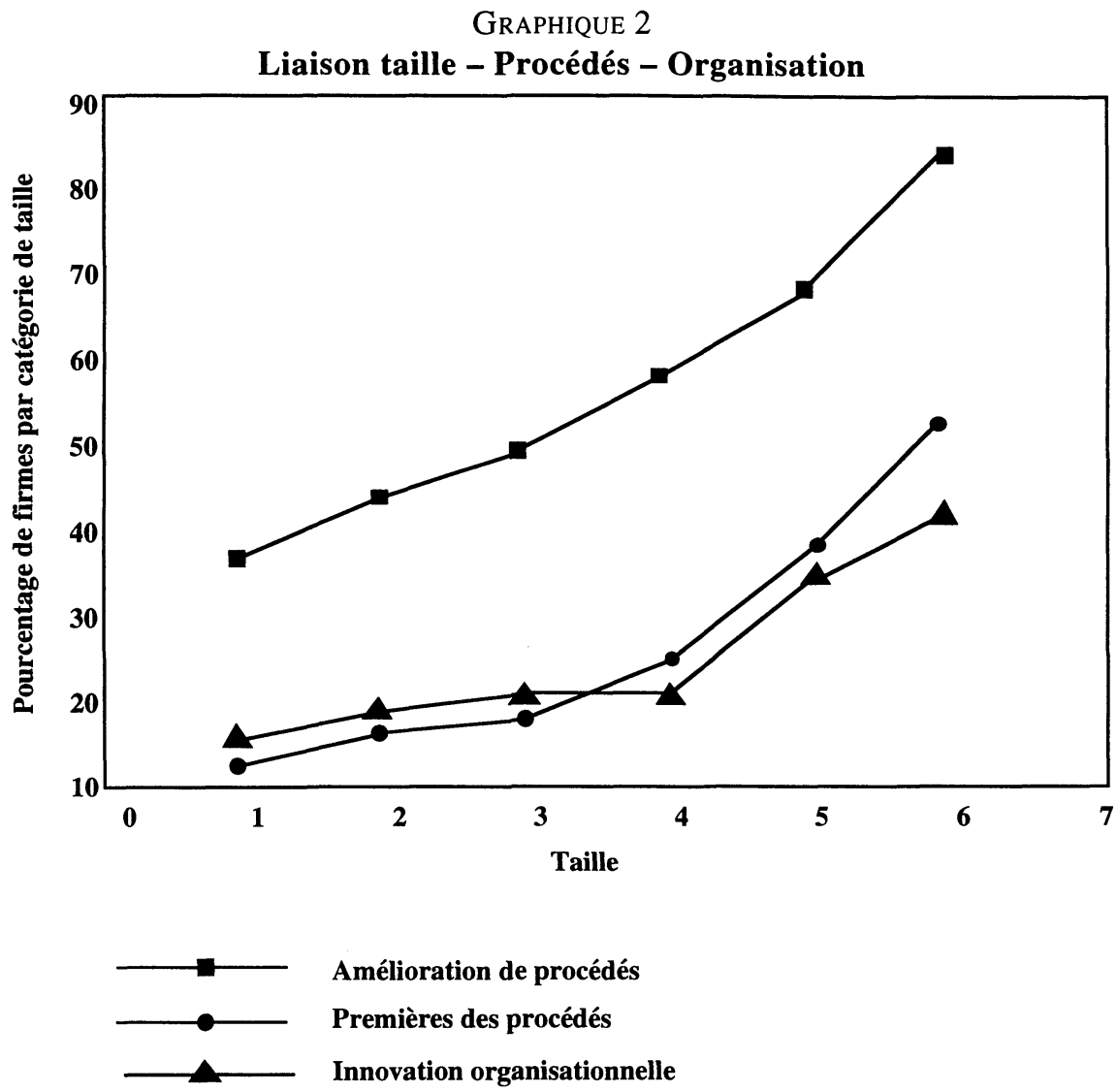

disparités intersectorielles. L'idée de Winter est largement corroborée par l'étude réalisée par Acs et Audretsch (1988), qui montrent que l'innovation répond, dans les PME, à des contraintes technologiques et économiques différentes de celles des grandes firmes. On constate ainsi aux États-Unis que dans certains secteurs, tels que l'optique ou les instruments de mesure, ce sont les petites firmes qui contribuent le plus à l'innovation (Audretsch, 1992), contrairement à des industries telles que l'aviation, par exemple.

Parallèlement, plusieurs études (Acs et Audretsch, 1991) montrent que les grandes firmes souffrent également de désavantages en matière de R-D, en particulier en ce qui concerne l'existence de déséconomies d'échelle liées à la production d'innovations. Les grandes firmes posséderaient un avantage en R-D en raison de la taille supérieure de leur output et donc de la possibilité d'appliquer leurs résultats sur une plus grande échelle, mais elles seraient également naturellement conduites à investir dans des projets moins risqués 
conduisant à améliorer leur technologie plutôt qu'à la bouleverser, ce qui n'est pas toujours le cas des petites firmes (Rosen, 1991). Plus récemment enfin, Cohen et Klepper (1992) introduisent une distinction entre les dépenses de R-D consacrées aux innovations de produits et de procédés et montrent que les firmes de plus grande taille font plus d'innovations de procédé. Pour leur part, White et al. (1988) observent que les petites firmes se distinguent tout particulièrement par leur capacité à s'engager dans l'innovation de produits.

Le cas français montre que la taille des firmes n'a pas de réelle influence sur la gamme des innovations entreprises. Les PMI comme les grandes firmes jouent sur toutes les possibilités d'innovation. La seule différence notable semble se situer au niveau de la qualité de l'innovation: les firmes de petite taille se révélant un peu moins présentes au niveau des premières de produits et de procédés (tableau 2), ainsi que le montre bien le cas des entreprises de moins de 50 salariés.

De manière plus générale, un examen de la littérature consacrée aux firmes de petite taille révèle que l'on s'accorde le plus souvent à considérer que ces dernières possèdent un certain nombre d'avantages en matière $d$ 'innovations telles que leur capacité à réagir rapidement à des changements provenant de la demande, leur rapidité interne de communication ou leur dynamique entrepreneuriale résultant de l'absence de bureaucratie. En revanche, elles sont réputées présenter plusieurs handicaps dans ce domaine, qu'il s'agisse de l'absence de ressources humaines qualifiées, de la difficulté à accéder aux ressources financières, de l'impossibilité à atteindre les économies d'échelle, de l'inadéquation avec les systèmes de brevets ou encore de leur lenteur à s'adapter aux réglementations publiques. Il faut, à ce sujet, se montrer très circonspect dans l'analyse des résultats des enquêtes, ainsi que le souligne Kleinknecht plus particulièrement : ce n'est pas parce qu'il n'y a pas de département de R-D dans une entreprise de petite taille que cette dernière ne se livre à aucune activité de R-D, la faiblesse des effectifs en R-D étant souvent partiellement compensée par un effort d'innovation important et par des stratégies de coopération.

Si de nombreuses PMI participent au processus d'innovation et de R-D, il est nécessaire (au-delà du nombre important de firmes présentes) de mesurer l'intensité de cet effort. En effet, on peut s'interroger sur la part que les entreprises manifestant une activité innovatrice consacrent aux dépenses de R-D ou aux activités d'innovation. Il faut ici cependant introduire de manière préalable une distinction qui concerne la mesure de l'activité innovatrice, la capacité d'innovation d'une firme pouvant être mesurée de manière différente par des indicateurs de nature très diverse. On trouve généralement quatre modalités de mesure de l'innovation qui présentent des caractéristiques diverses: les balances de paiements technologiques, les statistiques de R-D, 
les statistiques de brevets et les décomptes du nombre d'innovations. Il faut rappeler brièvement leurs spécificités (Archibugi, 1988): les statistiques de R-D (dépenses et personnel) donnent une bonne idée de l'effort (quantitatif, mais non qualitatif) réalisé par les firmes en matière d'innovation; mais aucune liaison linéaire ne met en relation cet input avec la production de savoir technologique, lequel peut également trouver sa source dans les activités de production quotidienne (innovations incrémentales, par exemple). Les statistiques de brevets permettent au contraire d'avoir une idée de l'output de technologie, mais elles souffrent de deux limites importantes: toutes les innovations ne sont pas brevetées, tant s'en faut, surtout les plus incrémentales, et il est impossible de distinguer entre un brevet recouvrant une innovation d'un poids très important et une autre d'un poids plus faible. Enfin, les relevés directs du nombre d'innovations présentent les mêmes avantages et souffrent évidemment de la même limite de repérage que les brevets : comment distinguer une «grosse» innovation d'une «petite».

Devant cette difficulté à mesurer l'intensité innovatrice, deux critères ont été retenus ici. Parmi les firmes se déclarant innovantes, on a choisi de sélectionner les firmes leaders. Sont considérées comme telles les firmes qui estiment être très bien placées du point de vue de l'innovation dans leur secteur d'activité par rapport à leurs principaux concurrents et qui s'orientent plus particulièrement vers la production de produits nouveaux pour le marché (tableau 4) (Bernard, 1994). Une deuxième mesure concerne les seules firmes ayant une activité de R-D. L'intensité de l'effort de recherche est alors caractérisée par deux ratios: dépenses internes de R-D/chiffre d'affaires et effectif de chercheurs/effectif total (tableau 5).

Tableau 4

Poids des leaders en matière d'innovation par classe de taille sur la population totale des firmes

(France, 1985-1990)

\begin{tabular}{l|c|c}
\hline \multicolumn{1}{c|}{ Taille } & $\begin{array}{c}\text { Nombre de firmes } \\
\text { dans la population totale } \\
\text { (en \% colonne) }\end{array}$ & $\begin{array}{c}\text { Nombre } \\
\text { de leaders } \\
\text { (en \% ligne) }\end{array}$ \\
\hline $20-49$ & 39 & 6 \\
$50-99$ & 21 & 9 \\
$100-199$ & 13 & 10 \\
$200-499$ & 13 & 13 \\
PMI & 86 & 19 \\
$500-1999$ & 10 & 29 \\
$>2000$ & 4 & \\
Total & $\mathbf{1 0 0}$ & \\
\hline
\end{tabular}

Source : LATAPSES-OST, enquête innovation SESSI. 
Les résultats sont très clairs. La grande taille favorise le leadership en matière d'innovation technologique. Ainsi, parmi les firmes de taille supérieure à 2000 salariés, $29 \%$ se considèrent comme des leaders dans leur activité d'innovation, alors que seules $8 \%$ des PMI estiment se trouver dans la même situation.

En revanche, et comme le montre le tableau 5, si l'on mesure l'intensité de la R-D, en termes de dépenses ou d'effectifs, la relation entre taille de la firme et intensité est inverse. Les petites firmes qui déclarent une activité de R-D consacrent proportionnellement beaucoup plus de moyens à cette activité relativement à leur taille.

\section{TABLEAU 5}

Intensité de la R-D par classe de taille

(France, 1990)

\begin{tabular}{l|c|c|c}
\hline Taille & $\begin{array}{c}\text { Ratio : dépenses } \\
\text { internes de R-D } \\
\text { sur CAHT } \\
\text { (en \% ligne) }\end{array}$ & $\begin{array}{c}\text { Ratio : effectifs } \\
\text { chercheurs sur } \\
\text { effectif total } \\
\text { (en \% ligne) }\end{array}$ & $\begin{array}{c}\text { Nombre moyen } \\
\text { de chercheurs }\end{array}$ \\
\hline $20-\quad 49$ & 24 & 14 & $(3$ à 7$)$ \\
$50-\quad 99$ & 10 & 7 & $(3$ à 7$)$ \\
$100-199$ & 6 & 3 & $(3$ à 6) \\
$200-499$ & 3 & 2 & $(4$ à 10) \\
$500-1999$ & 3 & 1 & $(5$ à 20) \\
$>2000$ & 3 & 1 & $(>20)$ \\
\hline
\end{tabular}

Source : LATAPSES, enquête recherche MERS.

Si l'incidence de la taille est nette quel que soit le critère retenu, innovation ou R-D, la spécificité sectorielle joue également, ainsi que le montre le tableau 6 . En effet, les secteurs considérés traditionnellement comme très dépendants de la technologie se caractérisent, dans le cas français, par une intensité plus grande de R-D. Là encore, les PMI rejoignent les grandes firmes, hormis quelques différences secondaires. Par exemple, dans l'aérospatiale, l'intensité de la recherche est beaucoup plus affirmée pour les grandes firmes que pour les PMI. C'est le cas inverse qui se présente pour les industries électroniques et informatiques, ainsi que dans les transports terrestres, où les PMI ont une intensité en R-D plus élevée, ces résultats confirmant largement ceux obtenus par Acs et Audretsch (1992) dans le cas américain. 
TABLEAU 6

Intensité de la R-D par grands secteurs

(France, 1990)

\begin{tabular}{|c|c|c|c|c|}
\hline \multirow[t]{2}{*}{ Secteurs } & \multicolumn{2}{|c|}{$\begin{array}{c}\text { Nombre de firmes } \\
\text { ayant une activité de R-D } \\
\text { (en \% colonne) }\end{array}$} & \multicolumn{2}{|c|}{$\begin{array}{c}\text { Intensité de la R-D } \\
\text { Dépenses internes de R-D/CAHT } \\
\text { (en \% ligne) }\end{array}$} \\
\hline & $\begin{array}{l}\text { Toutes } \\
\text { firmes }\end{array}$ & PMI & $\begin{array}{l}\text { Toutes } \\
\text { firmes }\end{array}$ & PMI \\
\hline Aérospatiale & 2 & 1 & 20 & 10 \\
\hline $\begin{array}{l}\text { Électronique } \\
\text { et informatique }\end{array}$ & 19 & 22 & 11 & 15 \\
\hline Pharmacie & 9 & 9 & 8 & 9 \\
\hline Biens/Équipement & 25 & 27 & 3 & 4 \\
\hline Transports terrestres & 5 & 4 & 3 & 7 \\
\hline Chimie/Plastiques & 16 & 17 & 3 & 4 \\
\hline Autres industries & 24 & 20 & 1 & 4 \\
\hline Total & 100 & 100 & & \\
\hline
\end{tabular}

Source : LATAPSES-OST, données enquête recherche MERS.

TABLEAU 7

Poids des leaders innovateurs par grands secteurs

(France, 1985-1990)

\begin{tabular}{l|cc|cc}
\hline Secteurs & \multicolumn{2}{|c|}{$\begin{array}{c}\text { Nombre de firmes } \\
\text { (en \% colonne) }\end{array}$} & \multicolumn{2}{c}{$\begin{array}{c}\text { Leaders par grands secteurs } \\
\text { (en \% ligne) }\end{array}$} \\
& $\begin{array}{l}\text { Toutes } \\
\text { firmes }\end{array}$ & PMI & $\begin{array}{l}\text { Toutes } \\
\text { firmes }\end{array}$ & PMI \\
\hline Aérospatiale & 1 & 1 & 18 & 15 \\
Électronique & 11 & 11 & 21 & 19 \\
$\quad$ et informatique & 4 & 3 & 25 & 21 \\
Pharmacie & 26 & 27 & 12 & 11 \\
Biens/Équipement & 3 & 3 & 10 & 5 \\
Transports terrestres & 12 & 12 & 11 & 5 \\
Chimie/Plastiques & 43 & 43 & 5 & \\
Autres industries & $\mathbf{1 0 0}$ & $\mathbf{1 0 0}$ & & \\
Total & & & & \\
\hline
\end{tabular}

Source : LATAPSES-OST, données enquête recherche MERS.

Ainsi que le montre le tableau 7, la présence de firmes qui s'estiment être des leaders dans leurs secteurs d'activité respectifs dépend de la nature de cette activité. Cependant, en pourcentage du total de la population étudiée des firmes innovantes, il existe peu de différences entre grandes entreprises 
et PME (partie gauche du tableau, \% colonne). Par contre, si l'on considère chaque secteur en particulier, les PME leaders sont un peu moins nombreuses (partie droite du tableau, \% ligne). Ici encore, la différence en matière d'innovation entre firmes de grande et de petite taille est peu significative.

D'un point de vue plus général, on constate donc que :

- l'effet taille joue un rôle prépondérant en matière d'innovation dans le cas de l'industrie française ;

- l'effet sectoriel est également important, l'intensité innovatrice se révélant différente selon les industries;

- il n'existe pas de spécificité de l'effet taille à l'intérieur des secteurs étudiés.

\section{Les caractéristiques dynamiques de l'activité innovatrice des PMI}

Une fois étudiées les relations entre la taille des entreprises françaises, la R-D et leur activité innovatrice, il faut se pencher tout spécialement sur les caractéristiques dynamiques de l'effort d'innovation. On doit en effet s'interroger sur le processus qui entraîne les PMI à avoir une activité innovatrice, qu'il s'agisse des motivations de la mise en place d'un processus de développement technologique, des sources de l'innovation, des liens entre la $R-D$ et l'innovation ou encore du cheminement par lequel les entreprises de petite taille sont conduites à entreprendre ou à poursuivre une activité relevant du domaine de l'innovation.

À la base de ce type de recherche réside la distinction entre différentes catégories de mesure du changement technologique. On a en effet, faute d'autres données disponibles, utilisé pendant longtemps les dépenses de R-D comme la seule approximation possible de l'intensité technologique. Dans les années 80 sont apparues de nouvelles sources de données, avec la disponibilité dans de nombreux pays des statistiques de brevets et licences, qui peuvent être considérées comme une mesure alternative de l'activité des firmes. Enfin, le calcul du nombre d'innovations produites par les entreprises et mises sur le marché (Pavitt, 1984) constitue une dernière catégorie de mesure de l'output en matière de technologie, même si elle souffre évidemment des mêmes limites que l'indicateur précédent.

Une fois établie la disponibilité de ces différents indicateurs se pose la question de la relation entre dépenses de R-D au sens large ou achats de brevets et activité innovatrice. Il faut en effet tout d'abord, dans la recension 
des sources de l'innovation, s'interroger sur l'importance des dépenses de R-D et leur influence sur une activité d'innovation ultérieure. Bien que peu de recherches aient été réalisées sur ce terrain, le travail effectué par Acs et Audretsch (1988) apporte un certain nombre d'éléments précieux quant au comportement des firmes américaines de petite taille. Ces auteurs trouvent en effet que, sur leur échantillon, l'augmentation de l'output d'innovations (le nombre d'innovations) est corrélée positivement avec celle des dépenses de R-D industrielle.

Par ailleurs, il est nécessaire d'introduire une distinction sur le type de financement de la R-D, selon que celle-ci est financée par des fonds publics ou à partir de capitaux privés. Griliches (1986) avait trouvé que le financement privé a une incidence plus forte sur l'activité innovatrice. Ce résultat est confirmé au niveau des PME par Acs et Audretsch (1991): ils montrent que la corrélation entre les dépenses privées de R-D et l'activité innovatrice est plus élevée que celle existant entre la R-D totale (les dépenses publiques et privées) et l'activité innovatrice.

Il semble qu'une des raisons de cette liaison réside dans le fait que les PME, comme d'autres firmes d'ailleurs (Cohen et Levinthal, 1989), utilisent une part importante d'innovations de leur cru et font largement appel à leurs capacités d'expertise propres en matière de technologie. Ce résultat renvoie d'ailleurs à deux présupposés. Le premier concerne les caractéristiques mêmes des innovations, lesquelles ne constituent pas un bien homogène et peuvent présenter des spécificités tout à fait différentes selon qu'il s'agit de grandes ou de petites firmes. Ainsi les PME vont souvent, en raison de leur faible taille, concentrer leurs efforts sur des innovations incrémentales trop modestes pour intéresser une grande corporation (Audretsch, 1992). Le deuxième présupposé est lié au coût d'adoption du savoir-faire extérieur à la firme, qui est toujours important, si bien que les PME désirant acquérir du savoir-faire technologique doivent auparavant développer de la R-D maison afin d'améliorer leurs capacités d'expertise et de développement. Ce résultat semble, dans le cas des entreprises de petite taille en tous les cas, être souvent le fruit d'un travail en petits groupes à l'intérieur même de la firme. Il n'exclut toutefois pas la mise en réseau, qui apparaît comme l'un des éléments centraux de la «nouvelle donne» industrielle et constitue la voie prioritaire d'acquisition de savoir-faire qu'il faudra ensuite s'approprier par un processus interne d'apprentissage. Ces réseaux sont de différents types puisqu'ils concernent aussi bien les rapports de sous-traitance avec des entreprises de taille plus importante, les relations de coopération au niveau de la R-D ou les collaborations dans le domaine des techniques de commercialisation que les échanges d'informations intervenant pendant le processus de 
production. Les nouveaux produits ou procédés résultent alors de ces échanges, quand ils ne sont pas développés conjointement par le biais de relations de coopération technologique. Ainsi que le suggère Dodgson (1990), on peut penser que dans ce cas, la base de l'activité innovatrice de ces petites firmes réside dans l'exploitation du savoir externe de réseau plus que dans la création d'un savoir propre.

Dans l'ensemble, on s'accorde souvent à reconnaître que les PME, qui apparaissent statiquement peu entreprenantes, le sont bien davantage lorsqu'on se situe dans une vision dynamique de leur activité (Audretsch, 1992). En effet, leur poids intrinsèque individuel se révèle plutôt faible par rapport à celui des grandes firmes, ne serait-ce qu'en ce qui concerne les seules dépenses de R-D, donnant ainsi une image quelque peu dévalorisante de leur activité. Il en va autrement en revanche quand on considère leur activité dans l'introduction de nouveaux produits ou procédés, même s'il s'agit le plus souvent d'innovations que l'on peut qualifier d'incrémentales. Ces innovations semblent souvent fondées sur la capacité à mobiliser les ressources humaines internes de la firme, en jouant en particulier sur sa flexibilité dans le processus de développement et sur sa capacité à n'accorder qu'une partie de son temps aux questions relevant de l'activité innovatrice (White et al., 1988).

La question des sources de l'innovation est donc fondamentale. Il faut cependant rappeler, avant toute étude appliquée, que «la population des firmes innovantes va bien au-delà de la minorité d'entreprises qui font de la R-D» (Auzeby et Francois, 1992), même si la R-D constitue l'un des facteurs principaux de l'activité innovatrice. L'enquête innovation menée par le SESSI a ainsi montré que les principales sources de l'innovation sont de nature interne, représentant un savoir-faire que les firmes ne peuvent occulter si elles veulent innover : c'est le cas pour $85 \%$ des firmes déclarant avoir eu une activité d'innovation dans les cinq années précédant l'enquête. Ces ressources internes englobent de la R-D interne, mais également l'activité d'étude et de méthode et les brevets dont la firme est titulaire. Dans l'ordre, le recours à l'activité d'étude (77\% des firmes innovantes) est plus fréquent que l'exploitation de la R-D interne (50\% des firmes innovantes) et l'utilisation de brevets dont la firme est titulaire ( $28 \%$ des firmes innovantes). De nombreuses firmes innovantes $(63 \%)$ font cependant appel à des sources externes d'innovation, telles que la R-D externe à l'entreprise (achetée à d'autres entreprises, à des organismes de recherche et à l'étranger : 46\%), la R-D interne au groupe (dans le cas où la firme en question est filiale d'un groupe : $28 \%$ ) et l'achat de licences et brevets (21\%) (Auzeby et Francois, 1992). 
Si $85 \%$ des firmes innovantes ont recours à au moins un type de ressource interne, une dichotomie apparaît cependant entre les PME et les grandes entreprises. On considère, dans le graphique 3, les seules firmes qui présentent les deux caractéristiques suivantes : être innovatrices et utiliser de manière intensive les sources internes d'innovation. On constate que les grandes firmes ont plus fréquemment recours à la R-D interne que les petites qui, elles, privilégient plutôt les activités d'étude et de méthode, sans pour autant avoir une préférence marquée pour l'une de ces sources d'innovation.

GRAPHIQUe 3

\section{Liaison taille - Sources internes innovation}
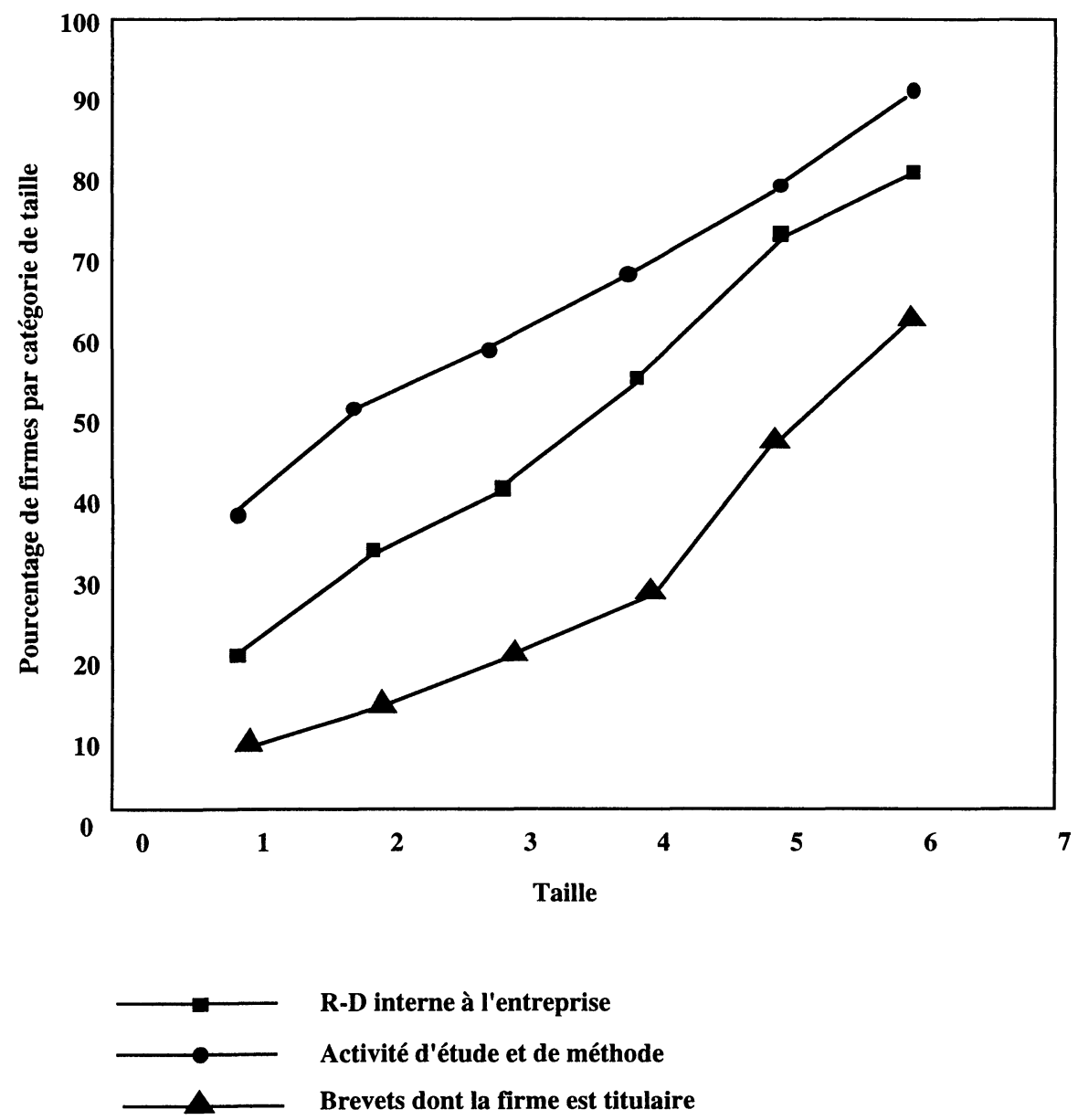

R-D interne à l'entreprise

Activité d'étude et de méthode

Brevets dont la firme est titulaire 
GRAPHIQUE 4

Liaison taille - Sources externes innovation

Fort contenu en...

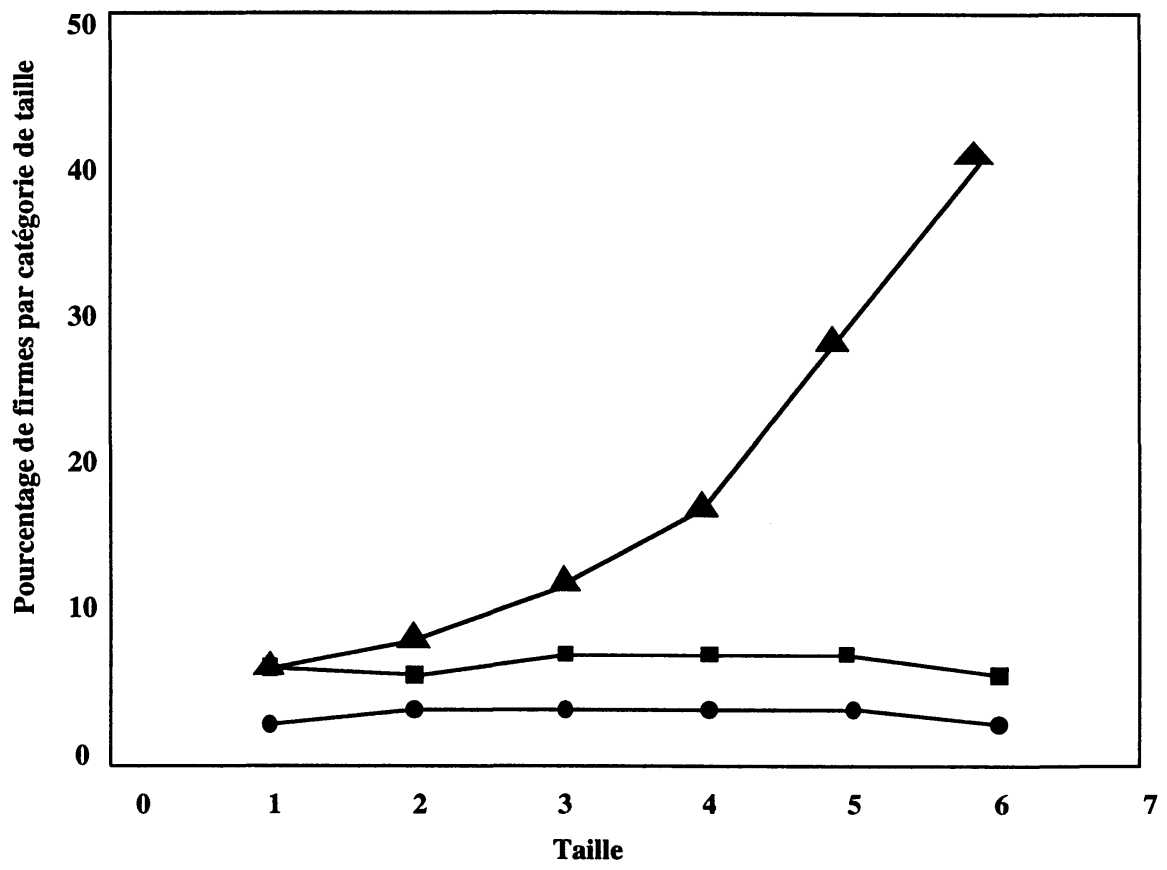

$\longrightarrow$ R-D externe à l'entreprise

$\longrightarrow$ Droits et licences

\section{R-D interne au groupe}

Ainsi que le montre le graphique 4, pour les firmes innovantes et utilisant de manière intensive des ressources externes, il existe peu de différence en fonction de la taille en ce qui concerne la R-D externe à l'entreprise et l'achat de droits et licences. Il n'en va pas de même pour la diffusion de la $\mathrm{R}-\mathrm{D}$ interne au groupe : elle se révèle beaucoup plus forte à mesure que la taille des firmes croît. Ce résultat est probablement dû en partie au fait que peu de firmes de petites tailles sont rattachées à des groupes.

Pouvoir innover suppose que l'on dispose des ressources technologiques nécessaires, mais cela ne préjuge en aucun cas d'une perpétuation de l'activité d'innovation dans le temps. Une entreprise peut en effet avoir besoin d'innover pendant une certaine période, puis s'arrêter. Pour répondre 
à cette question dans le cas français, il serait nécessaire de disposer d'un test portant sur une période suffisamment longue et conduisant à juger de la permanence de l'innovation. L'enquête innovation ne le permet pas. Cependant, cette enquête incorporait une question sur l'introduction d'innovations par les entreprises pour la période 1990-1995. Le tableau 8 montre que les firmes sont restées relativement prudentes sur ce sujet: si une entreprise non innovante sur cinq a l'intention d'innover dans le futur, le même nombre décide de ne plus renouveler l'expérience. On constate à nouveau l'existence d'un lien entre la taille des firmes et l'intention d'innover, qui est plus faible pour les PMI. Ce lien provient sans doute de ce que l'expérience acquise en matière d'innovation reste un facteur décisif pour envisager la poursuite de celle-ci (la proportion de petites firmes innovantes étant plus faible, un pourcentage moins important est conduit à envisager l'avenir de manière optimiste) (Bernard, 1994).

TABleau 8

Intention d'innover et taille des firmes

(France 1991-1995)

\begin{tabular}{l|c|c|c|c}
\hline Taille & $\begin{array}{c}\text { Nombre de firmes } \\
\text { ayant l'intention } \\
\text { d'innover } \\
\text { dans le futur } \\
\text { (en \% ligne) }\end{array}$ & Produits & Procédés & $\begin{array}{c}\text { (en \% ligne) } \\
\text { Organisation } \\
\text { technologique }\end{array}$ \\
(en \% ligne) & (en \% ligne) \\
\hline $20-49$ & 56 & 37 & 43 & 36 \\
$50-99$ & 63 & 47 & 52 & 44 \\
$100-199$ & 70 & 55 & 61 & 51 \\
$200-499$ & 76 & 63 & 68 & 54 \\
PMI & 60 & 43 & 51 & 41 \\
GE & 87 & 79 & 82 & 70 \\
\hline
\end{tabular}

Source : LATAPSES-OST, données enquête innovation SESSI.

Si l'on rentre dans les détails, il apparaît tout d'abord que les firmes adoptent des attitudes différentes suivant le type d'innovation: prudentes quant aux innovations de produit, elles se tournent plutôt vers des innovations de procédés. En réalité, leur démarche se révèle plus complexe à l'examen, même si les comportements de prudence sont généraux. La caractéristique majeure est en effet l'émergence de l'innovation organisationnelle: alors que peu de firmes (19\%) avaient innové en matière d'organisation sur la période passée (1985-1990), $41 \%$ prévoient de le faire dans le futur, le plus souvent en combinaison avec des innovations de procédés. Ainsi, il semble bien que 
la nécessité de mettre en place de nouvelles formes d'organisation liées au changement technologique soit une contrainte nouvelle pour les firmes françaises (Greenan et al., 1993). On peut cependant partager les entreprises innovantes en deux catégories distinctes en fonction de leur optique. Les firmes qui se considèrent comme des leaders dans leurs secteurs envisagent directement de futures innovations de produits, alors que celles qui se considèrent comme moins bien positionnées préfèrent effectuer un détour par l'innovation organisationnelle (Bernard, 1994).

\section{Conclusion}

Cette étude avait pour objet l'analyse de la situation des PMI françaises en matière d'activité innovatrice, en se fondant sur les déterminants principaux utilisés pour ce type de recherche dans d'autres pays. Alors que chacun s'accorde à dire aujourd'hui que la compétitivité internationale d'une nation dépend en grande partie du rôle joué par les PMI, nos résultats montrent que l'activité innovatrice des PMI françaises est importante, et même croissante sur une période récente. Ces petites firmes, au-delà de la diversité de leurs situations, sont plus rarement innovantes que les grandes entreprises. Toutefois, quand elles manifestent une activité innovatrice, leurs comportements restent largement comparables à ceux des grandes firmes, l'effet taille jouant peu.

Il apparaît, par ailleurs, dans notre étude que les PMI innovatrices sont rarement des leaders en matière d'innovation et qu'elles se caractérisent également par une plus grande turbulence dans le domaine de l'activité de R-D. Afin de leur permettre de maintenir une activité innovatrice, il semble souhaitable que la politique de recherche et technologie, qui a surtout par le passé privilégié les grandes firmes, rééquilibre son action en faveur des PMI en tenant compte de leurs besoins spécifiques.

\section{Bibliographie}

ACs, Z. et D.B. AudretsCH (1988), «Innovation in large and small firms: an empirical analysis », American Economic Review, vol. 78, ${ }^{\circ} 4$, p. 678-689.

ACs, Z. et D.B. AUdretsch (1991), Innovation and Small Firms, Cambridge, Mass., MIT Press.

ARChIbUgI, D. (1988), "Alla ricerca di una misurazione utile dell' innovazione tecnologica», l'Industria, vol. IX, n², p. 231 et 264. 
AUdRETSCH, D.B. (1992 ), «Small business in industrial economics: the new learning » Communication présentée à la $7^{\mathrm{e}}$ session de l'École d'été d'économie industrielle Cargèse, septembre.

AUZEBY, F. et J.P. FrANCOIS (1992), «L'innovation technologique dans l'industrie», le 4 Pages, SESSI, $n^{\circ} 101$, janvier.

BERNARD, J. (1994), «Les représentations de l'innovation des firmes françaises, un lien entre le passé et le futur», dans l'Innovation technologique dans l'industrie, les chiffres clés, SESSI, Paris, Dunod.

CARLSSON, B.G. (1989), «The evolution of manufacturing technology and its impact on industrial structure: an international comparison», Small Business Economics, vol. 1, p. 21-38.

COHEN, W.D. et S. KLEPPER (1992), «Firm size and the nature of innovation within industries : the case of process and product R-D», Communication présentée à l'International Schumpeter Association, Kyoto, août.

COHEN, W.D., R.C. LEVIN et D.C. MOWERY (1987), «Firm size and R-D intensity : a re-examination », Journal of Industrial Economics, vol. XXXV, $\mathrm{n}^{\circ} 4$, p. 543-565.

Cohen, W.D. et D.A. LEvinthal (1989), «Innovation and learning: the two faces of R-D », Economic Journal, vol. 99, p. 569-596.

CREmer, J. et M. Sirbu (1978), «Une analyse économétrique de l'effort de R-D de l'industrie française », Revue économique, vol. 29, n 5, p. 940-954.

DodGson, M. (1990), «Technology strategy in small and medium-sized firms », dans Z. Acs et D.B. Audretsch (dir.), The Economics of Small Firms, Londres, Kluwer.

Greenan, N., D. Guellec, G. Broussaudier et L. Miotti (1993), «Innovation organisationnelle, dynamisme technologique et performances des entreprises », Document de travail, Direction des études et synthèses économiques, INSEE.

GRILICHES, Z. (1986), «Productivity, R-D and basic research at the firm level in the 1970's », American Economic Review, p. 141-154.

Kamien, M.I. et N.L. SChWARTZ (1982), Market Structure and Innovation, Cambridge, Cambridge University Press.

KLEINKNECHT, A. (1987), «Measuring R-D in small firms : how much are we missing ? », Journal of Industrial Economics, vol. XXXVI, p. 253-256.

Levin, R., A. KLEVorick, R.R. NELSON et S. WinTER (1987), «Appropriating the returns from industrial R-D», Brookings Papers on Economic Activity, p. 783-820.

OST (1993), Science et Technologie: Indicateurs 1994, Rapport de l'OST, Paris, Economica. 
PARANQUe, B. (1992), Contraintes Économiques des PMI et des Grandes Entreprises 1981-1990, Les études de la Centrale des bilans, Banque de France, Paris.

PAVITT, K. (1984), «Patterns of technological change : towards a taxonomy and a theory », Research Policy.

PAVITT, K., M. RoBSON et J. TOWNSEND (1987), « The size distribution of innovating firms in the U.K., 1945-1983 », Journal of Industrial Economics, vol. XXXV, $\mathrm{n}^{\circ} 3$, p. 297-316.

Piore, M.J. et C.F. SABel (1984), The Second Industrial Divide: Possibilities for Prosperity, New York, Basic Books.

Rosen, P.J. (1991), «Research and development with asymetric firm sizes », Rand Journal of Economics, vol. 22, p. 411-429.

SCHERER, F.M. (1984), Innovation and Growth: Schumpeterian Perspectives, Cambridge, Mass., MIT Press.

SChumaCher, E.F. (1978), Small is Beautiful, Paris, Seuil.

SCHUMPETER, J.A. (1954), Capitalisme, socialisme et démocratie, Paris, Payot.

SESSI (1991), Les chiffres clefs des PMI, Paris, Dunod.

White, M., H.J. BraczyK, A. Ghobadian et J. Niebuhr (1988), Small Firms' Innovation, Why Regions Differ, Policy Studies Institute, Iver, Bucks, Bourne Offset Ltd.

WINTER, S.G. (1984), «Schumpeterian competition in alternative technological regimes », Journal of Economic Behavior and Organization, vol. 5, p. 287-320. 